\title{
Vasostatin increases oxygenation of B16-F10 melanoma tumors and raises therapeutic efficacy of cyclophosphamide
}

\author{
Tomasz Cichoń ${ }^{\bowtie}$, Magdalena Jarosz, Ryszard Smolarczyk, Barbara Ogórek, \\ Sybilla Matuszczak, Marek Wagner, Iwona Mitrus, Aleksander Sochanik, \\ Joanna Jazowiecka-Rakus and Stanisław Szala
}

\section{Center for Translational Research and Molecular Biology of Cancer, Maria Skłodowska-Curie Memorial Cancer Center and Institute of Oncology,} Gliwice Branch, Gliwice, Poland

\begin{abstract}
One of the preconditions of effective anticancer therapy is efficient transfer of the therapeutic agent (chemotherapeutic) to tumor cells. Fundamental barriers making drug delivery and action difficult include underoxygenation, elevated interstitial pressure, poor and abnormal tumor blood vascular network and acidic tumor milieu. In this study we aimed at developing an optimized scheme of administering a combination of an angiogenesis-inhibiting drug (vasostatin) and a chemotherapeutic (cyclophosphamide) in the therapeutic treatment of mice bearing experimental B16-F10 melanoma tumors. We report that the strongest tumor growth inhibition was observed in mice that received two, three or four vasostatin doses in combination with one injection of cyclophosphamide (i.e., V2 + CTX, V3 + CTX or V4 + CTX schemes). Double administration of vasostatin increases oxygenation of B16-F10 tumors. On the other hand, its five-fold administration lowers tumor oxygenation, breaks down tumor vascular network (increasing hypoxia) and leads in consequence to death of cancer cells and appearance of necrotic areas in the tumor. A decreased cyclophosphamide dose in combination with two doses of vasostatin (V2 + CTX scheme) inhibits tumor growth similarly to a larger dose of cyclophosphamide alone.
\end{abstract}

Key words: vasostatin, cyclophosphamide, oxygenation, normalization of tumor blood vessels, anticancer therapy

Received: 26 March, 2012; revised: 17 July, 2012; accepted: 29 August, 2012; available on-line: 03 September, 2012

\section{INTRODUCTION}

Abnormal tumor blood vascular network and poor penetration of cancer cells by drugs are basic causes of low efficacy of most anticancer strategies Jain, 2001; Cairns et al., 2006; Minchinton \& Tannock, 2006; Vaupel, 2006; Fukumura \& Jain, 2007). According to Jain et al. (2004) improved functioning of tumor vasculature, the so-called normalization of tumor blood vessels, may substantially affect accessibility of drugs to cancer cells (Tong et al., 2004; Goel et al., 2012). The phenomenon is based on reestablishing the equilibrium between proangiogenic and antiangiogenic factors thanks to which tumor blood vessels become functionally similar to normal vessels (Jain, 2005; Carmeliet \& Jain, 2011).

Antiangiogenic drugs, when administered according to a specific scheme, allow achieving the so-called "normalization window". Only during the presence of such a window is it possible to observe better oxygenation of tumor tissue (and decreased hypoxia) as well as lowered intratumoral pressure (Tong et al., 2004; Jain, 2005; Matsumoto et al., 2011). The "normalization window" is a short-term episode lasting no longer than a few days. At its conclusion tumor blood vessels start reverting to the conditions prior to normalization.

We here aimed at finding out an optimum scheme of administering an antiangiogenic-acting protein (vasostatin) in combination with cyclophosphamide, a wellknown chemotherapeutic. As a therapeutic model we chose experimental B16-F10 malignant melanoma tumors borne by $\mathrm{C} 57 \mathrm{Bl} / 6$ mice. Vasostatin is the $\mathrm{N}$-terminal domain of calreticulin, a protein found inside the endoplasmatic reticulum. In vivo, its activity inhibits angiogenesis whereas in vitro inhibition of proliferation of endothelial cells stimulated by basic fibroblast growth factor (bFGF) or vascular-endothelial growth factor (VEGF) has been observed (Jazowiecka-Rakus et al., 2006; Cai et al., 2008; Sun et al., 2008).

The strongest inhibition of tumor growth was obtained applying a combination of two, three or four vasostatin administrations with one of dose cyclophosphamide. Other combinations turned out to be less effective, since further administration of vasostatin caused destruction of tumor cells and formation of necrotic areas. As a result, High-Mobility Group Box-1 Protein (HMGB1) is released into the extracellular space. This protein participates in the formation and growth of tumor blood vessels which, in turn, leads to rapid tumor regrowth (Smolarczyk et al., 2010).

We decided to use vasostatin in our experiments as we had used this protein in our earlier investigations. This study is a follow-up of the experiments carried out by Jazowiecka-Rakus et al. (2007). They confirmed that the combination of these two drugs was superior compared to either drug administered alone. We here decided to verify the possibility of developing a therapeutic scheme that would further improve the effect that had been observed before.

\section{MATERIALS AND METHODS}

Cell culture. Murine melanoma B16-F10 cells (ATCC) were propagated in RPMI 1640 supplemented with 10\%

\footnotetext{
$\triangle$ e-mail: tcichon@io.gliwice.pl
}

Abbreviations: V, vastatin; CTX, cyclophosphamide; B16-F10, murine melanoma cells; bFGF, basic fibroblast growth factor; VEGF, vascular-endothelial growth factor; HMGB1, High-Mobility Group Box-1 Protein; PBS', physiological buffered saline; RPMI 1640, cell culture medium. 
fetal bovine serum (FBS). Cell culture was maintained in a standard $37^{\circ} \mathrm{C} / 5 \% \mathrm{CO}_{2}$ incubator. Cells were passaged every 2-3 days.

Animals. Mice (6- to 8-week-old C57Bl/6 females) were from own animal facility. Consent for work with animals was obtained from the local Ethics Commission (Silesian Medical University in Katowice).

Isolation and purification of vasostatin. Recombinant MBP/vasostatin fusion protein (vasostatin) was obtained and purified using pMAL System (Protein Fusion and Purification System, New England BioLabs, MA, USA). Isolation and purification was done essentially according to (Jazowiecka-Rakus et al., 2006).

Therapy of B16-F10 tumor-bearing mice using a combination of vasostatin with cyclophosphamide. Mice had their left dorsal side shaved and were then inoculated subcutaneously with B16-F10 cells $\left(2 \times 10^{5} / 100 \mu \mathrm{L} \mathrm{PBS}^{-} /\right.$animal). Experimental therapy was started when tumors reached ca. $65 \mathrm{~mm}^{3}$ (7 to 8 days following inoculation with cancer cells). Vasostatin was injected intratumorally using $60 \mu \mathrm{g} /$ mouse $/ 100 \mu \mathrm{L}$ FB solution (Formulation Buffer consisted of sterile saline solution containing 5\% human albumin and $0.5 \%$ mannitol) (Jazowiecka-Rakus et al., 2006). Experimental animal groups $(n=5)$, differing by the number of vasostatin doses applied, were set up (V1-V5). Subsequent vasostatin injections were performed at 24-hour intervals. Single doses of cyclophosphamide (CTX) were injected peritoneally (100 or $170 \mathrm{mg} / \mathrm{kg}$ body mass) 24 hours following the last vasostatin administration (see Browder et al., 2000). Tumor growth was evaluated daily using caliper measurements. Tumor volumes were calculated using the formula: $\mathrm{V}\left[\mathrm{mm}^{3}\right]=($ width $) 2 \times($ length $) \times 0.52$.

Determination of hypoxic regions in B16-F10 murine melanoma tumors following therapy with vasostatin. Throughout this series of experiments 60 $\mu \mathrm{g}$ of vasostatin/mouse (in $100 \mu \mathrm{L}$ FB) was injected intratumorally, typically on the 6th day following inoculation of animals with B16-F10 cells when the tumor volume reached ca. $65 \mathrm{~mm}^{3}$. Determination of hypoxic regions was accomplished using HypoxyprobeTM-1 kit (Chemicon, CA, USA), in accordance with the manufacturer's protocol. One hour prior to material collection animals were given intravenous injection of pimonidazole $(60 \mathrm{mg} / \mathrm{kg}$ body weight). Material from five tumors obtained from every experimental group was excised, cut and stained. The excised tumor tissue was fixed for 24 hours in $2 \%$ paraformaldehyde $(\mathrm{pH}=7.2)$, paraffin-embedded, cut into 5-6 $\mu \mathrm{m}$-thick sections and stained with HypoxyprobeTM-1 and counterstained with hematoxylin-eosin. The range of hypoxia-characteristic colors was determined arbitrarily on the basis of DAB staining intensity, using a positive reference section. Due to size constraints, each section was divided into several observation fields (57 ), which were then photographed (Nikon Eclipse 80i fluorescence microscope, $40 \times$ magnification). Hypoxia was quantified with the analySISD software. Ratios of hypoxic phase to the sum of hypoxic and non-hypoxic phases (the sum was assumed to equal the section surface area) obtained for particular fields were averaged. Tumor hypoxia was calculated from the ratios obtained from 6-8 sections.

Immunohistochemical identification of HighMobility Group Box-1 Protein (HMGB1). Paraffin-embedded B16-F10 tumor sections were deparaffinized, hydrated, and incubated in $0.3 \% \mathrm{H}_{2} \mathrm{O}_{2}$. Antigen retrieval was achieved by boiling in citrate buffer

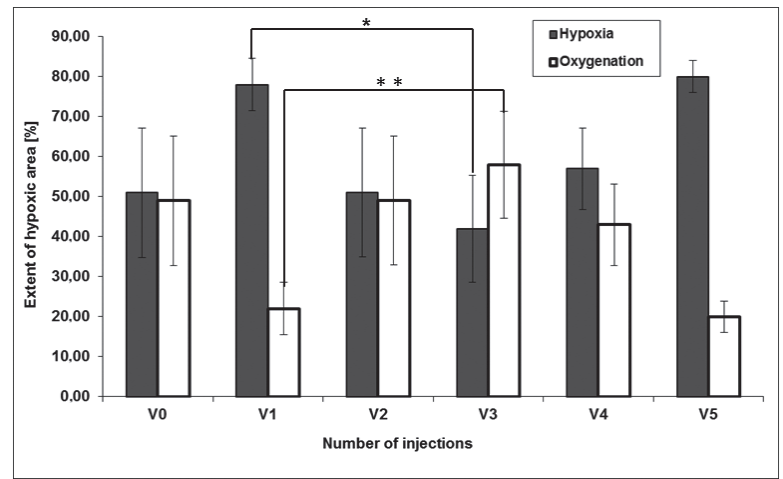

Figure 1. Effect of vasostatin on the extent of hypoxia in B16F10 tumors.

Tumor hypoxic regions were assessed using HypoxyprobeTM-1 kit (see: Materials and Methods). Vasostatin administration was started six days after inoculation of mice with B16-F10 cells, when tumor volumes reached ca. $65 \mathrm{~mm}^{3}$. Six experimental groups $(\mathrm{n}=5$ animals) were set up. Twenty-four hours following last vasostatin administration the mice were sacrificed and tumor material excised. Bars show percentage proportions of hypoxic and oxygenated tumor areas. The sum of both bars corresponds to total surface area $(100 \%)$ of the examined tumor sections. Bar data are averages from 6-8 sections obtained from different regions of two separate tumors (for details see: Materials and Methods). The number of vasostatin injections (e.g., V3) is given. ${ }^{*} p<0.007$ hypoxia compared in groups V1 and V3 Tukey post hoc analysis after ANOVA. ** $p<0.007$ oxygenation compared in groups $\mathrm{V} 1$ and V3 Tukey post hoc analysis after ANOVA.

(10 mM; pH 6.0), whereas nonspecific binding was blocked with $2.5 \%$ horse serum. The sections were incubated at RT for 1 hour with rabbit anti-HMGB1 primary antibody $(0.7 \mathrm{mg} / \mathrm{mL}$; diluted 1:100, Abcam) and with horse anti-rabbit secondary antibody conjugated to horseradish peroxidase (HRP; EC 1.11.1.7) from ImmPRESSTM REAGENT Anti-Rabbit Ig kit (Vector). In order to obtain the final colored product the preparations were incubated with 3-diaminobenzidine (DAB) from ImmPACTTM DAB kit. The enzymatic reaction yielded a brown-colored product. The preparations were then dehydrated and mounted with VectaMountTM medium.

Statistical analysis. Differences between groups were determined by applying ANOVA followed by the Tukey post hoc test. A $p$-value lower than 0.05 was considered statistically significant.

\section{RESULTS}

\section{Effect of vasostatin and CTX administration on the extent of B16-F10 tumor oxygenation}

The improvement in tumor oxygenation was dependent on the number of vasostatin injections performed. The appearance of increased oxygenation areas was accompanied by a concomitant decrease of hypoxic areas. After the second vasostatin administration the oxygenation of tumor tissue rose from $21 \%$ (V1) to $49 \%$ (V2), and to $55 \%$ (V3) following three administrations of the drug. Five administrations of vasostatin (V5) resulted in decreased oxygenation, down to $17 \%$ (Fig. 1). The strongest inhibition of tumor growth was observed when two, three or four vasostatin injections were combined with one dose of cyclophosphamide (V2+CTX; V3+CTX or V4+CTX schemes) (Fig. 2). 


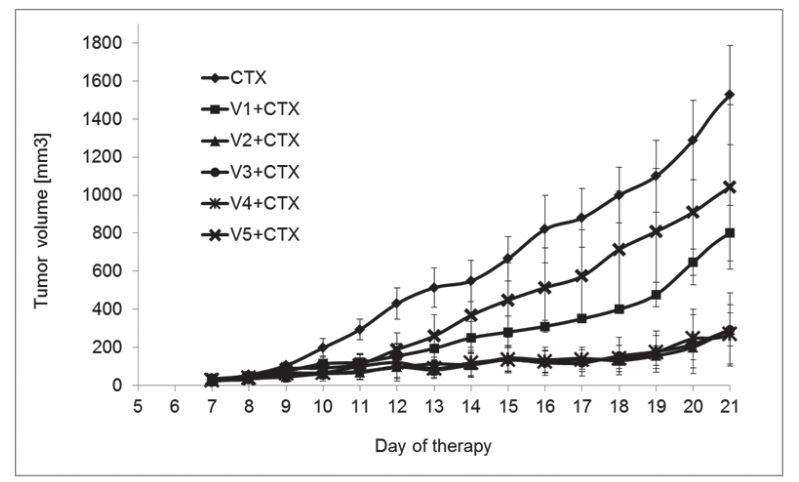

Figure 2. Effect of various vasostatin-cyclophosphamide combinations on growth of B16-F10 murine melanoma tumors.

Vasostatin administration was started six days after inoculation of C57Bl/6 mice with B16-F10 cells, when tumor volumes reached ca. $65 \mathrm{~mm}^{3}$. Experimental groups $(n=5)$, differing by the number of vasostatin doses applied, were set up (V1-V5). Five different vasostatin-cyclophosphamide combinations were evaluated. Vasostatin doses were injected intratumorally $(60 \mu \mathrm{g} / \mathrm{mouse} / 100 \mu \mathrm{L}$ FB). Subsequent vasostatin injections were performed at 24-hour intervals. Single doses of cyclophosphamide (CTX) were injected peritoneally $(170 \mathrm{mg} / \mathrm{kg}$ body mass). The graph shows tumor volumes on the corresponding day of therapy. Each data point on the graph represents averaged volume of five tumors. Numerals denote the number of vasostatin administrations, e.g., V4 means four injections. Graphs depicting survival curves for untreated as well as vasostatin alone-treated mice can be found in JazowieckaRakus et al. (2007).

\section{Effect of repeated vasostatin administration on the structure of B16-F10 tumors}

Administration of five consecutive doses of vasostatin causes destruction of tumor blood vessels, death of cancer cells and appearance of necrotic domains within tumors. As a result of these processes, HMGB1 (HighMobility Group Box-1 Protein) protein is released into the intercellular space (Fig. 3).

\section{Effect of V2+CTX vasostatin-cyclophosphamide combination at low CTX dose (100 mg/kg body weight) on B16-F10 tumor growth}

When administered vasostatin in combination $(60 \mu \mathrm{g} /$ animal $/ 100 \mu \mathrm{L} \mathrm{FB}$ ) with cyclophosphamide at $100 \mu \mathrm{g} / \mathrm{g}$ body weight, B16-F10 tumor growth was inhibited to the same extent as cyclophosphamide alone but at a substantially higher dose $(170 \mu \mathrm{g} / \mathrm{g}$ body weight). With the higher cyclophosphamide dose, combination therapy with vasostatin gave, however, the best therapeutic effects (Fig. 4).

\section{DISCUSSION}

The most frequently observed effect of antitumor therapies is only partial elimination of cancer cells. This severe limitation of efficacy of current strategies is due to the specific structure and the physico-chemical properties of the tumor milieu, and/or to drug resistance of cancer cells. An abnormal structure of the tumor blood vessels and dysfunction of lymphatic vessels lead to increased intratumoral pressure inhibiting transfer of chemotherapeutics as well as leaking of the interstitial fluid (containing the drug) outside of the tumor. The low $\mathrm{pH}$ of the extracellular matrix (acidification of tumor microenvironment due to anaerobic metabolism of cancer cells) may inhibit or even inactivate drug action (Cairns et al., 2006). Drug resistance can be elicited by overex-

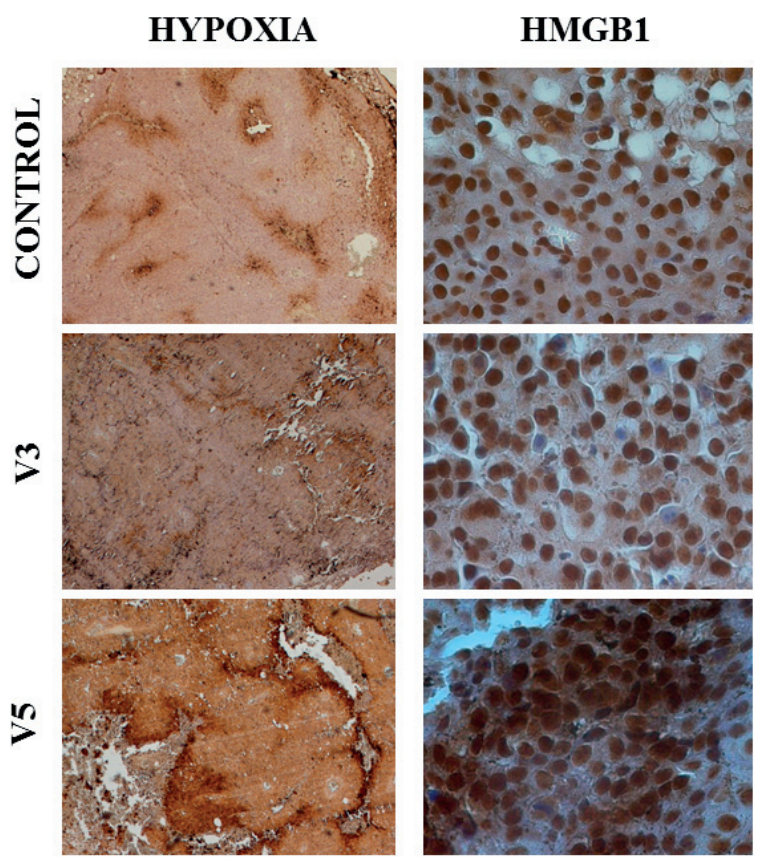

Figure 3. Effect of subsequent vasostatin doses on the structure of B16-F10 murine melanoma tumors.

For experimental details see Fig. 1. Micrographs show: tumor hypoxic areas (brown); HMGB1 protein (brown) in cells (control, V3) and in extracellular space (V5). Magnification: 4x (hypoxia); 20x (HMGB1).

pression of proteins involved in chemotherapeutics' removal from cells or proteins involved in repair of DNA damage that occurred in the course of therapy (Martinez-Lacaci et al., 2007).

Single-drug anticancer therapies have mostly proven unsuccessful. Much better effects can be obtained with multidrug therapies or approaches combining varying therapeutic strategies (e.g., chemotherapy combined with radiotherapy). The goal of our study was to establish the best therapeutic scheme of administering a combination of two drugs (vasostatin and cyclophosphamide) in the treatment of mice bearing experimental B16-F10 malignant melanoma tumors.

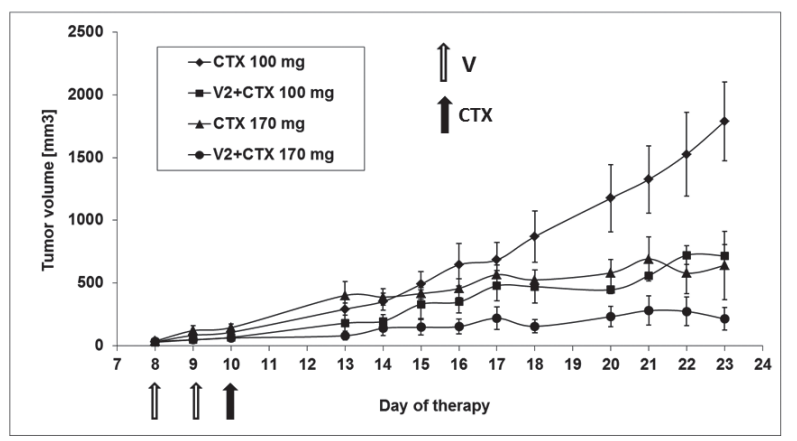

Figure 4. Effect of administering V2 + CTX combination on B16F10 tumor growth.

Vasostatin administration was started six days after inoculation of mice with B16-F10 cells, when tumor volumes reached ca. $65 \mathrm{~mm}^{3}$. Vasostatin doses were injected intratumorally $(60 \mu \mathrm{g} /$ mouse/100 $\mu \mathrm{L} F B)$. Subsequent vasostatin injections were performed at 24-hour intervals. Single doses of cyclophosphamide (CTX) were injected peritoneally (100 or $170 \mathrm{mg} / \mathrm{kg}$ body mass). Each data point represents averaged tumor volume $( \pm S$.D.) from five animals. 
The study is a follow-up of experiments carried out by Jazowiecka-Rakus et al. (2007). It was then confirmed that the combination of these two drugs was superior compared to either drug administered alone. We here decided to verify whether it was possible to develop a therapeutic scheme that would further improve the effect observed previously. We also attempted to investigate how vasostatin enhanced the therapeutic effect obtainable with cyclophosphamide as well as the impact of vasostatin on tumor tissue oxygenation. We observed that, depending on the amount of vasostatin delivered, the extent of oxygenated tumor areas is variable. Following single vasostatin injection the oxygenated area dwindled from $50 \%$ (control group) to $21 \%$ in the investigated experimental tumor sections. A single injection of vasostatin is not enough to normalize the blood vessels and the higher level of hypoxia is due to tumor growth. After two or three vasostatin administrations the extent of oxygenated area rose, reaching ca. $55 \%$, only to retreat to $17 \%$ after five administrations.

In the experiments that followed vasostatin therapy was complemented with cyclophosphamide, which was administered 24 hours after the final vasostatin injection. It was observed that two-, three- or four-fold injection of vasostatin ( $60 \mu \mathrm{g} /$ mouse) following single injection of cyclophosphamide (170 $\mu \mathrm{g} / \mathrm{g}$ body mass), i.e., the V2 + CTX or V3 + CTX or V4 + CTX administration schemes effectively inhibited tumor growth. A similar growth arrest was not observed for single (V1 + C'TX scheme) or five-fold vasostatin administration (V5 + CTX scheme). Five injections of vasostatin destroyed the tumor blood vessels, caused death of neoplastic cells and brought the appearance of necrotic areas within the tumor (hypoxia) (Fig. 3). As a result of these processes HMGB1 protein was released into the extracellular space (Fig. 3). This protein is partly responsible for the formation and growth of new tumor blood vessels and, ultimately, for rapid tumor regrowth.

The growth inhibition of tumors treated with various combinations of vasostatin and cyclophosphamide is linked to an increased oxygenation of the tumor tissue. Well-oxygenated regions were detected in sections of tumor tissue derived from mice treated with two, three or four injections of vasostatin. No such increase could be detected in tumor material obtained from mice treated with one or five injections of vasostatin. On the other hand, in tumors from control mice treated with cyclophosphamide alone, in which well-oxygenated tumor regions are comparable to those in tumors from mice treated with two doses of vasostatin, no tumor growth inhibition was detected. Thus, not only an increased tumor tissue oxygenation can enhance therapeutic efficiency of cyclophosphamide, but also the cytotoxic effect of vasostatin could be at play.

Angiogenesis inhibitors, which prevent formation of new blood vessels also cause, according to Jain (2005), the occurrence of the so-called "normalization window". Such a window denotes a short period during which morphological properties of tumor blood vessels become improved; among other things, the pericyte coverage of blood vessels is enhanced, basement membrane is formed, the density of vessels increases and they become less leaky (Carmeliet \& Jain, 2010; Matsumoto et al., 2011; van Baumagarten et al., 2011; Ribatti, 2011). Their diameter is also reduced (Tong et al., 2004; Winkler et al., 2004). Owing to these changes, conditions appear within the tumor that are conducive towards access of drugs to cancer cells (Tong et al., 2004; Jain, 2005; Chabot et al., 2011; Goel et al., 2011; 2012).

It is commonly assumed that this is the best moment to commence chemo- or radiotherapy (Kozin et al., 2001; Jain, 2005; Segars et al., 2006; Goel et al., 2012). An improved tumor oxygenation was observed in our study about 24 hours after the second vasostatin administration and lasting for the next two days. Our results show some similarity to those obtained by Segara et al. (2006) who used thalidomide to normalize tumor vasculature. In our opinion vasostatin, similar to the CD101 antibody or thalidomide, may lead to the appearance of a "normalization window" leading to normalized tumor vasculature.

Vasostatin administered in a suitable combination with cyclophosphamide enhances its therapeutic efficacy. The chemotherapeutic injected at a dose of $100 \mathrm{mg} / \mathrm{kg}$ body mass, when complemented with vasostatin injections $(2 \times 60 \mu \mathrm{g} /$ mouse), yields therapeutic results comparable to those of cyclophosphamide at a dose of $170 \mathrm{mg} / \mathrm{kg}$ body mass.

In the presented experiments we used B16-F10 murine melanoma tumor model since in our earlier studies we also used that model. Unfortunately, it is a very fastgrowing tumor and we had to commence therapeutic experiments when the tumor size did not exceed about 65 $\mathrm{mm}^{3}$ in order to generate meaningful data. In the nearest future we plan to carry out similar experiments using an orthotopic GL261 murine glioma tumor model. We believe that this model is perfectly suitable for antiangiogenic studies.

One should mention that vasculature normalization could affect therapy in both positive and negative way. The former effects include augmented efficacy of chemo- and radiotherapy while the latter include increased possibility of metastasis (Liu et al., 2011). In our experiments we did not observe formation of metastases; instead we noted an abrupt resumption of the tumor growth following cessation of vasostatin administration.

\section{TO CONCLUDE}

Since we have been unable to demonstrate undisputably the presence of vasculature normalization process by assessing, for example, pericyte coverage or increased vascular permeability, we can only suppose that vasostatin, an antiangiogenic protein, increases oxygenation of B16-F10 tumor tissue when administered in appropriate doses and sequence. This probably reflects normalization of the tumor vasculature and follows generation of the so-called "normalization window". While the window lasts, concurrent administration of cyclophosphamide enhances its effectiveness.

\section{Acknowledgements}

This work was supported by the Ministry of Science and Higher Education, grant No. N N401 018337.

\section{REFERENCES}

Browder T, Butterfield C, Kräling B, Shi B, Marshall B, O'Reilly M, Folkman J (2000) Antiangiogenic scheduling of chemotherapy improves efficacy against experimental drug-resistant cancer. Cancer Res 60: 1878-1886.

Cai KX, Tse LY, Leung C, Tam PK, Xu R, Sham MH (2008) Suppression of lung tumor growth and metastasis in mice by adenoassociated virus-mediated expression of vasostatin. Clin Cancer Res 14: 939-949. 
Cairns R, Papandreou I, Denko N (2006) Overcoming physiologic barriers to cancer treatment by molecularly targeting the tumor microenvironment. Mol Cancer Res 4: 61-70.

Carmeliet P, Jain RK (2011) Principles and mechanisms of vessel normalization for cancer and other angiogenic diseases. Nat Rev Drug Discov 10: 417-427.

Chabot S, Jabrane-Ferrat N, Bigot K, Tabiasco J, Provost A, Golzio M, Noman MZ, Giustiniani J, Bellard E, Brayer S, Aguerre-Girr M, Meggetto F, Giuriato S, Malecaze F, Galiacy S, Jaiis JP, Chose O, Kadouche J, Chouaib S, Teissié J, Abitbol M, Bensussan A, Le Bouteiller P (2011) A novel antiangiogenic and vascular normalization therapy targeted against human CD160 receptor. J Exp Med 208: 973-986.

Fukumura D, Jain RK (2007) Tumor microvasculature and microenvironment: Targets for anti-angiogenesis and normalization. Microvasc Res 74: 72-84.

Goel S, Duda DG, Xu L, Munn LL, Boucher Y, Fukumura D, Jain RK (2011) Normalization of the vasculature for treatment of cancer and other diseases. Physiol Rev 91: 1071-1121.

Goel S, Wong AH, Jain RK (2012) Vascular normalization as a therapeutic strategy for malignant and nonmalignant disease. Cold Spring Harb Perspect Med 2: a006486.

Jain RK (2003) Molecular regulation of vessel maturation. Nature Med 9: $685-693$.

Jain RK (2005) Normalization of tumor vasculature: an emerging concept in antiangiogenic therapy. Science 307: 58-63.

Jain RK, Duda D, Clark J, Loeffler J (2006) Lessons from phase III clinical trials on anti-VEGF therapy for cancer. Nature Clinical Practice 3: 24-39.

Jazowiecka-Rakus J, Jarosz M, Kozłowska D, Sochanik A, Szala S (2007) Combination of vasostatin and cyclophosphamide in the therapy of murine melanoma tumors. Acta Biochim Pol 54: 125-133.

Kozin S, Boucher Y, Hicklin D, Bohlen P, Jain R, Suit H (2001) Vascular endothelial growth factor receptor-2-blocking antibody potentiates radiation-induced long-term control of human tumor xenografts. Cancer Res 61: 39-44.

Liu J, Liao S, Huang Y, Samuel R, Shi T, Naxerova K, Huang P, Kamoun W, Jain RK, Fukumura D, Xu L (2011) PDGF-D Improves drug delivery and efficacy via vascular normalization, but promotes lymphatic metastasis by activating CXCR4 in breast cancer. Clin Cancer Res 170: 3638-3648.

Martinez-Lacaci I, Garcia Morales P, Soto JL, Saceda M (2007) Tumour cells resistance in cancer therapy. Clin Transl Oncol 9: 13-20.
Matsumoto S, Batra S, Saito K, Yasui H, Choudhuri R, Gadisetti C, Subramanian S, Devasahayam N, Munasinghe JP, Mitchell JB, Krishna MC (2011) Antiangiogenic agent sunitinib transiently increases tumor oxygenation and suppresses cycling hypoxia. Cancer Res 71: 6350-6359.

Minchinton AI, Tannock IF (2006) Drug penetration in solid tumours. Nat Rev Cancer 6: 583-592.

Normalization, But Promotes Lymphatic Metastasis by Activating CXCR4 in Breast Cancer. Clin Cancer Res 17: 3638-3648.

Ribatti D (2011) Vascular normalization: a real benefit? Cancer Chemother Pharmacol 68: 275-278.

Segers J, Di Fazio V, Ansiaux R, Martinive P, Feron O, Wallemacq P, Gallez B (2006) Potentiation of cyclophosphamide chemotherapy using the anti-angiogenic drug thalidomide: Importance of optimal scheduling to exploit the 'normalization' window of the tumor vasculature. Cancer Lett 20: 1-7.

Smolarczyk R, Cichoń T, Kamysz W, Głowala-Kosińska M, Szydło A, Szultka L, Sieroń AL, Szala S (2010) Anticancer effects of CAMEL peptide. Lab Invest 90: 940-952.

Sun Q, Xu Q, Dong X, Cao L, Huang X, Hu Q, Hua ZC (2008) A hybrid protein comprising ATF domain of pro-UK and VAS, an angiogenesis inhibitor, is a potent candidate for targeted cancer therapy. Int J Cancer 123: 942-950.

Tong R, Boucher Y, Kozin S, Winkler F, Hicklin D, Jain RK (2004) Vascular normalization by vascular endothelial growth factor receptor 2 blockade induces a pressure gradient across the vasculature and improves drug penetration in tumor. Cancer Res 64: 3731-3736.

Vaupel P (2006) Abnormal microvasculature and defective microcirculatory function of solid tumors. In V ascular-targeted Therapies in Oncology. Siemann DW, eds, pp 9-29. John Wiley \& Sons Ltd, Chichester, United Kingdom.

von Baumgarten L, Brucker D, Tirniceru A, Kienast Y, Grau S, Burgold S, Herms J, Winkler F (2011) Bevacizumab has differential and dose-dependent effects on glioma blood vessels and tumor cells. Clin Cancer Res 17: 6192-6205.

Winkler F, Kozin S, Tong R, Chae S, Booth M, Garkavtsew I, Xu L, Hicklin D, Fukumura D, Tomasa E. Munn L, Jain RK (2004) Kinetics of vascular normalization by VEGFR2 blockade governs brain tumor response to radiation: Role of oxygenation, angiopoietin-1, and matrix metalloproteinases. Cancer Cell 6: 553-563. 
